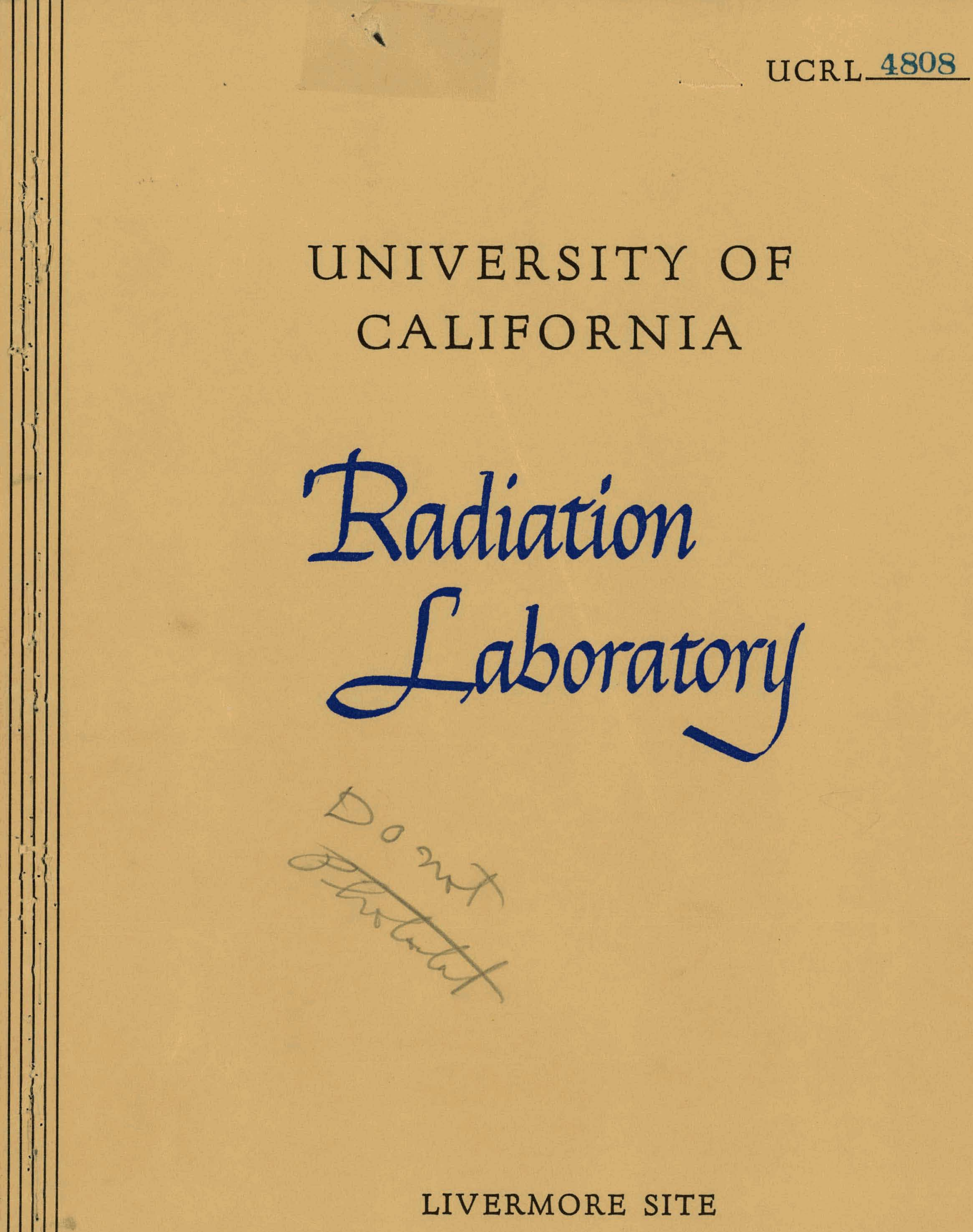




\section{DISCLAIMER}

This report was prepared as an account of work sponsored by an agency of the United States Government. Neither the United States Government nor any agency Thereof, nor any of their employees, makes any warranty, express or implied, or assumes any legal liability or responsibility for the accuracy, completeness, or usefulness of any information, apparatus, product, or process disclosed, or represents that its use would not infringe privately owned rights. Reference herein to any specific commercial product, process, or service by trade name, trademark, manufacturer, or otherwise does not necessarily constitute or imply its endorsement, recommendation, or favoring by the United States Government or any agency thereof. The views and opinions of authors expressed herein do not necessarily state or reflect those of the United States Government or any agency thereof. 


\section{DISCLAIMER}

Portions of this document may be illegible in electronic image products. Images are produced from the best available original document. 


\section{UNIVERSIT Y OF CALIFORNIA}

Radiation Laboratory, Livermore Site

Livermore, California

\section{THE APPLICATION OF PULSED NEUTRON SOURCES TO CRITICALITY MEASUREMENTS}

Lawrence Passell, Joel Bengston, and Donald C. Blair January 30, 1957

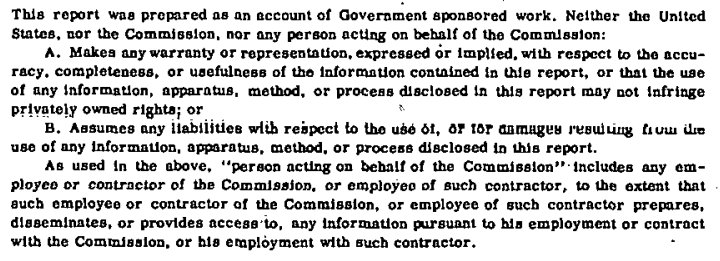

Printed.for the U.S. Atomic Energy Commisston 


\author{
THE APPLICATION OF PULSED NEUTRON SOURCES \\ TO CRITICALITY MEASÜREMENTS \\ Lawrence Passell, Joel Bengston, and Donald..C. Blair \\ University of California Radiation Laboratory \\ Livermore Site
}

January, 30,1957

\author{
ABSTRACT \\ i
}

Experimentally. measured prompt-neutron decay constants for subcritical. systems are of considerable value as normalization data for cal culations of prompteneutron time constants. A method of measuring these decay constants with a pulsed neutron source, neutron detector and multichannel time-delay analyzer has been developed and used on a number of fast-spectrum subcritical systems. An analysis of this method based on the one-energy group, one-delayed-neutron group reactor kinetic equations is presented and discussed. The curve, of neutron flux versus time predicted by the kinetic equations is compared with the observed curve obtained : with a $13.1-\mathrm{cm}$ diam. enriched-uranium sphere to indicate the application and limitations of the analysis. 


\title{
THE APPLICATION OF PULSED NEUTRON SOURCES TO CRITICALITY MEASUREMENTS*
}

Lawrence Passell, Joel Bengston, and Donald C. Blair

\author{
University of California Radiation Laboratory \\ Livermore Site
}

January 30,1957

The prompt-neutron time constants of fast.spectrum systems are of considerable importance in predicting the prompt critical behavior of these systems :

Theoretical estimates of prompt-neutron time constants do not generally require the solution of the time-dependent transport equation; in fact when delayed-neutron effects can be neglected, the problem is easily trans formed to the steady-state case. ${ }^{l}$ The calculation is then reduced to the simpler problem of solving a modified steady-state transport equation which for precise work, actually consists of a set of coupled equations representing the various neutron energy groups. . This type of problem is amenable to numerical methods, in particular, the $S_{n}$ method developed by Carlson ${ }^{2}$ has been used successfully, in a wide variety of cases.

Experimentally measured values of prompt-neutron decay constants for subcritical systems provide a natural normalization for the se calculations. During the past year, therefore, considerable effort has been devoted to the development of a method for measuring prompt-neutron decay, constants with a pulsed neutron source. ${ }^{3}$

* This paper.was presented extemporaneously by L. Passell at the winter meeting (December 10-12, 1956) of the American Nuclear Society, held in Washington, D.C.

${ }^{1}$ B. Davison, Transport Theory of Neutrons, National Research Council of Canada report No. LiT-18.(1947), Chapter 1 , Section 2.

${ }^{2}{ }_{B} \cdot G$. Carlson, Solution of. The Transport Equation by $S_{n}$ Approximations, LA-1891 (1955).

${ }^{3}$ Passell, Abramson, Andre, and Perez-Mendes, Measurements on Subcritical Assemblies With a Pulsed.Neutron Source, UCRL-4673 (1956). 
In principle the method consists simply of introducing short.bursts of neutrons into subcritical systems and then observing the decay of the leakage neutron flux with a time-delay analyzer. If the system is sufficiently subcritical to be relatively unaffected by delayed-neutron. contributions and if transient effects due to the source are of short duration, the leakage flux will decay exponentially with a time constant

$$
a=\frac{1-k_{\text {eff }}}{\ell}
$$

which is the prompt-neutron decay constant.

The behavior of the leakage neutrons in a pulsed fast spectrum assembly is described approximately by the one-energy group, one-delayed-neutron group kinetic equations

$$
\begin{aligned}
& \frac{d n(t)}{d t}=\frac{k_{e f f}(1-\beta)-1}{\ell} n(t)+\lambda c(t)+S(t) \\
& \frac{d c(t)}{d t}=\frac{\beta k_{\text {eff }}}{\ell} n(t)-\lambda c(t)
\end{aligned}
$$

where $n(t)=$ total number of neutrons in the system

$$
\begin{aligned}
& c(t)=\text { total number of delayed neutron emitters in the system } \\
& \beta \quad=\text { fraction of fission neutrons that are delayed } \\
& \lambda \quad=\text { weighted average decay constant of the delayed neutron emitters } \\
& k_{\text {eff }}=\text { effective multiplication factor } \\
& \ell \quad=\text { average neutron lifetime } \\
& S(t)=\text { rate at which source neutrons are introduced into the system. }
\end{aligned}
$$

$S(t)$ varies with the type of pulsed neutron source used. As an approximation we will assume

$$
S(t)=\frac{S_{0}\left(e^{\gamma t}-1\right)}{e^{\gamma \tau}-1} \quad \text { when } .0 \leq t \leq \tau
$$




$$
S(t)=S_{0} e^{-\gamma(t-\tau)} \quad \text { when } t \geq \tau
$$

i.e., the rate at which source neutrons are introduced into the system increases exponentially with a time constant $y$ for a time $\tau$ and then decreases exponentially with the same time constant.

Equations (1) and (2) with $S(t)$ given by Eqs. (3a) and (3b) can be inte grated directly by applying the Laplace transform. The solutions for $n(t)$ and.c(t) are given in the Appendix. Considerable simplification in the form of the solutions is possible for fast spectrum systems. Since the purpose of the experiments is to measure prompt-neutron decay constants, $k_{e f f}$ is deliberately chosen small enough so that

$$
\frac{1-k_{\text {eff }}}{k_{\text {eff }}} \gg \beta, \text { hence } \frac{1-k_{\text {eff }}(1-\beta)}{\ell} \cong \frac{1-k_{\text {eff }}}{\ell}=a
$$

Neutron lifetimes in the systems of interest are typically of the order of $10^{-8}$ seconds, hence

$$
\frac{1-k_{e f f}(1-\beta)}{\ell} \gg \lambda \text {. }
$$

Also $\gamma$, the time constant of the source burst, which is determined by the characteristics of the pulsed neutron source, is typically very large compared to $\lambda$. Hence under the conditions of the experiment, both the decay time of the neutron burst and the decay time of the neutrons in the assembly is fast compared to the decay time of the delayed neutron emitters. With these restrictions, and noting that

$\dot{e}^{\gamma \tau} \gg 1$, the solutions reduce to

$$
\begin{aligned}
n(t) & \stackrel{\frac{S_{0} e^{-\gamma \tau}}{(\gamma+a)} e^{\gamma t}+}{\cong}\left[n(0)-\frac{\lambda c(0)}{a}+\frac{S_{0} \gamma e^{-\gamma \tau}}{a(\gamma+a)}\right] e^{-a t}+\frac{\lambda c(0)}{a} e^{-\lambda t} \\
& -\frac{S_{0}}{a} e^{-\gamma \tau} \text { when } 0 \leq t \leq \tau .
\end{aligned}
$$




$$
\begin{aligned}
& n(t) \cong\left[n(0) e^{-a \tau}-\frac{\lambda c(0)}{a} e^{-a \tau}+\frac{S_{0} \gamma e^{-(\gamma+a) \tau}}{a(\gamma+a)}+\frac{2 S_{0} \gamma}{\gamma^{2}-a^{2}}-\frac{S_{0}}{a} e^{-\gamma \tau}\right] e^{-a(t-\tau)} \\
& -\frac{S_{0}}{(\gamma-a)} e^{-\gamma(t-\tau)}+\frac{\lambda c(0)}{a} e^{-\lambda t} \quad \text { when } \quad \tau \leq t \\
& c(t) \cong\left[\frac{\beta k_{e f f}}{a l}+c(0)+\frac{S_{o} \beta k e f f}{a \lambda \ell} e^{-\gamma \tau}\right] e^{-\lambda t}+\frac{S_{o} \beta k}{e f f} e^{-\gamma \tau}\left[\frac{e^{\gamma t}}{\gamma(\gamma+a)}-\frac{1}{a \cdot \lambda}\right] \\
& -\frac{\beta k_{e f f}}{a l}\left[n(0)+\frac{S_{0} \cdot e^{-\gamma \tau}}{a(\gamma+a)}\right] e^{-a t} \quad \text { when } 0 \leq t \leq \tau \\
& c(t) \cong\left[\frac{\beta k_{e f f} n(0)}{a \ell} e^{-\lambda \tau}+c(0) e^{-\lambda \tau}+\frac{S_{o} \beta_{k} k_{e f f} e^{-\gamma \tau} \cdot\left(e^{-\lambda \tau}-1\right)}{a \lambda \ell}\right. \\
& \left.+\frac{2 S_{0} \beta k_{e f f}}{a \gamma l}\right] e^{-\lambda(t-\tau)}+\frac{S_{o} \beta k_{e f f}}{\gamma_{\ell} \ell(\gamma-a)} e^{-\gamma(t-\tau)} \\
& -\frac{\beta k_{e f f}}{\mid a l}\left[n(0) e^{-a \tau}+\frac{S_{0} \gamma e^{-(\gamma+a) \tau}}{a(\gamma+a)}+\frac{2 S_{q}^{\gamma}}{\gamma^{2}-a^{2}}-\frac{S_{0}}{a} e^{-\gamma \tau}\right] e^{-a(t-\tau)}
\end{aligned}
$$$$
\text { when } \quad \tau \leq \mathrm{t} \text {. }
$$

Since the neutron bursts are introduced into the assembly periodically, $n(0)$ and $c(0)$ can be evaluated by requiring that

$$
\begin{aligned}
& n(0)=n(T) \\
& c(0)=c(T)
\end{aligned}
$$


where $\mathrm{T}$ is the time interval between neutron bursts: ${ }^{4} \mathrm{~T}$ is chosen much. longer than 1/a but very short compared to $1 / \lambda$. Applying condition (6a) to Eqs. (4a) and (4b) and condition (6b) to Eqs. (5a) and (5b), we find

$$
\begin{gathered}
n(0)=\frac{\lambda c(0)}{a} e^{-\lambda T}+\ldots \\
c(0)=\frac{\beta k_{e f f} n(0)}{a l} e^{-\lambda T}+c(0) e^{-\lambda T}+\frac{S_{0} \beta k_{e f f} e^{-\gamma \tau}\left(e^{-\lambda \tau}-1\right)}{a \lambda l} e^{-\lambda(T-\tau)} \\
+\frac{2 S_{0} \beta_{j} k_{e f f}}{a \gamma l} \cdot e^{-\lambda(T-\tau)}+\ldots
\end{gathered}
$$

from which we can obtain the approximate expressions

$$
\begin{aligned}
& n(0) \cong \frac{2 S_{o} \beta k_{\text {eff }}}{\gamma a^{2} l T} \\
& c(0) \cong \frac{2 S_{o} \beta k_{\text {eff }}}{\gamma \lambda a l T} .
\end{aligned}
$$

Substituting back into Eqs. (4a) and (4b); we then have

$$
\begin{gathered}
n(L) \cong S_{0}\left[\frac{1}{\gamma+a} e^{\gamma(t-\tau)}-\frac{1}{a} e^{-\gamma \tau}+\frac{\gamma e^{-a t}}{(\gamma+a) a}+\frac{2 \beta k \text { eff }}{\gamma a^{2} l T}\right] \text { when } 0 \leq t \leq \tau . \\
n(t) \cong S_{0}\left\{\left[\frac{\gamma e^{-(\gamma+a) \tau}}{a(\gamma+a)}+\frac{2 \gamma}{\gamma^{2}-a^{2}}-\frac{e^{-\gamma \tau}}{a}\right] e^{-a(t-\tau)}-\frac{e^{-\gamma(t-\tau)}}{\gamma-a}+\frac{2 \beta k}{\gamma a^{2} \ell T}\right\} \\
\text { eff }
\end{gathered}
$$

${ }^{4}$ These conditions hold only after the concentrations of the delayed neutron emitters build up to their equilibrium values. 
Equations $(8 \mathrm{a})$ and $(8 \mathrm{~b})$ describe the periodic change in the number of neutrons in a subcritical fast. spectrum system under typical experimental conditions. To illustrate the application of these equations, consider a representative experiment.

$$
\begin{aligned}
& a=0.46 \times 10^{8} \text { seconds }^{-1} \\
& \gamma=5 \times 10^{8} \text { seconds }-1 \\
& \tau=5 \times 10^{-8} \text { seconds } \\
& T=4 \times 10^{-7} \text { seconds }
\end{aligned}
$$

Figure 1 shows a plot of $n(t)$ as predicted by Eqs. (8a) and (8b) (dark line) and, for comparison, $n(t)$ as experimentally observed for an unreflected enriched-uranium. sphere $13.1 \mathrm{~cm}$ in diameter (dotted line). In the experiment the neutron bursts introduced into the assembly consisted of 14-Mev neutrons produced by short bursts of deuterons striking the tritium-loaded target of a Cockcroft-Walton accelerator. The target.was located approximately one cm from the surface of the sphere. Leakage neutrons were detected by a Hornyak fast neutron detector placed close to the surface of the sphere diametrically opposite the accelerator target. A Los Alamos time-to-pulse height converter, triggered by, the deuteron burst.striking the target, was used.with a 50 -channel pulse-height analyzer as a time-delay analyzer.

Clearly Eqs. (8a) and $(8 b)$ represent the behavior of the system quite well except in the region where source transient effects are appreciable. Poor agreement in this region is to be expected for a number of reasons. Perhaps the most.important is that the one-energy group kinetic equations do not take into account the transition from a monoenergetic 14-Mev source neutron energy spectrum to a fissionspectrum. After the decay begins, however, the oneenergy group model is more satisfactory because the energy spectrum remains -relatively stationary. "Another reason why the agreement is not better in the transient region is that the kinetics equations as sume a normal mode space distribution of source neutrons. This obviously does not represent the true state of affairs in the experiment. Evidently a certain amount of diffusion will have to take place as the source space distribution changes to a normal mode distribution. These two considerations are probably responsible for most of the difficulty. Other inadequacies, such as the use of a single group of delayed 
neutrons, do not seriously affect the results since the time.scale of the decay is too short to be influenced by the details of delayed neutron emission.

Examination of Eqs. (8a) and $(8 \mathrm{~b})$ and of the experimental results indicates that during an appreciable part of the cycle the leakage flux decays exponentially. with a time constant

$$
a=\frac{1-k_{\text {eff }}}{\ell},
$$

consequently a can be measured with very reasonable accuracy. The major limitation in the method is the stability and linearity of the time-delay analyzing system. In practice we have been able to attain. 3-5\% precision in measured a's. With a permanent installation, the accuracy can probably be improved appreciably.

A considerable variety of measurements on bare enriched-uranium systems have been made and compared.with values calculated from a sixenergy group transport equation normalized originally to a Los Alamos critical mass experiment. The agreement. was within the experimental error in every case. ${ }^{5}$ As an indication of the sensitivity of the method, decay curves for a 13. 1-cm-diameter enriched-uranium sphere and an 11.9-cm sphere are plotted together in Fig. 2. The dashed line indicates the shape of the neutron burst. without the assembly in.place for comparison.

${ }^{5}$ L. Germain, Theoretical Analysis of. Time-Dependent. Characteristics of Fast Spectrum: Fissioning. Assemblies, paper presented at the second annual meeting of The American Nuclear Society, held in Chicago, Illinois, June $6-8,1956$. 


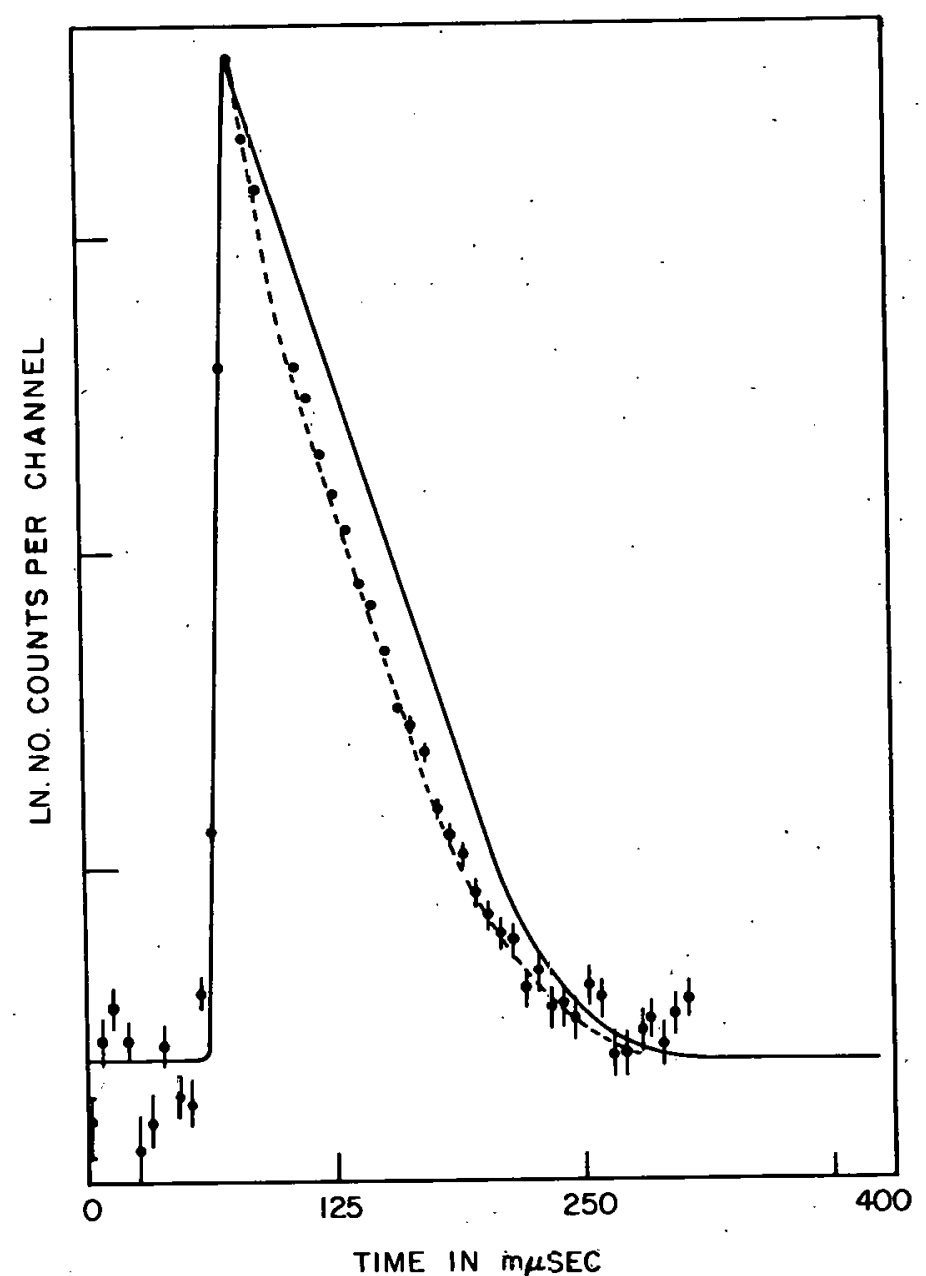

MUL .2603

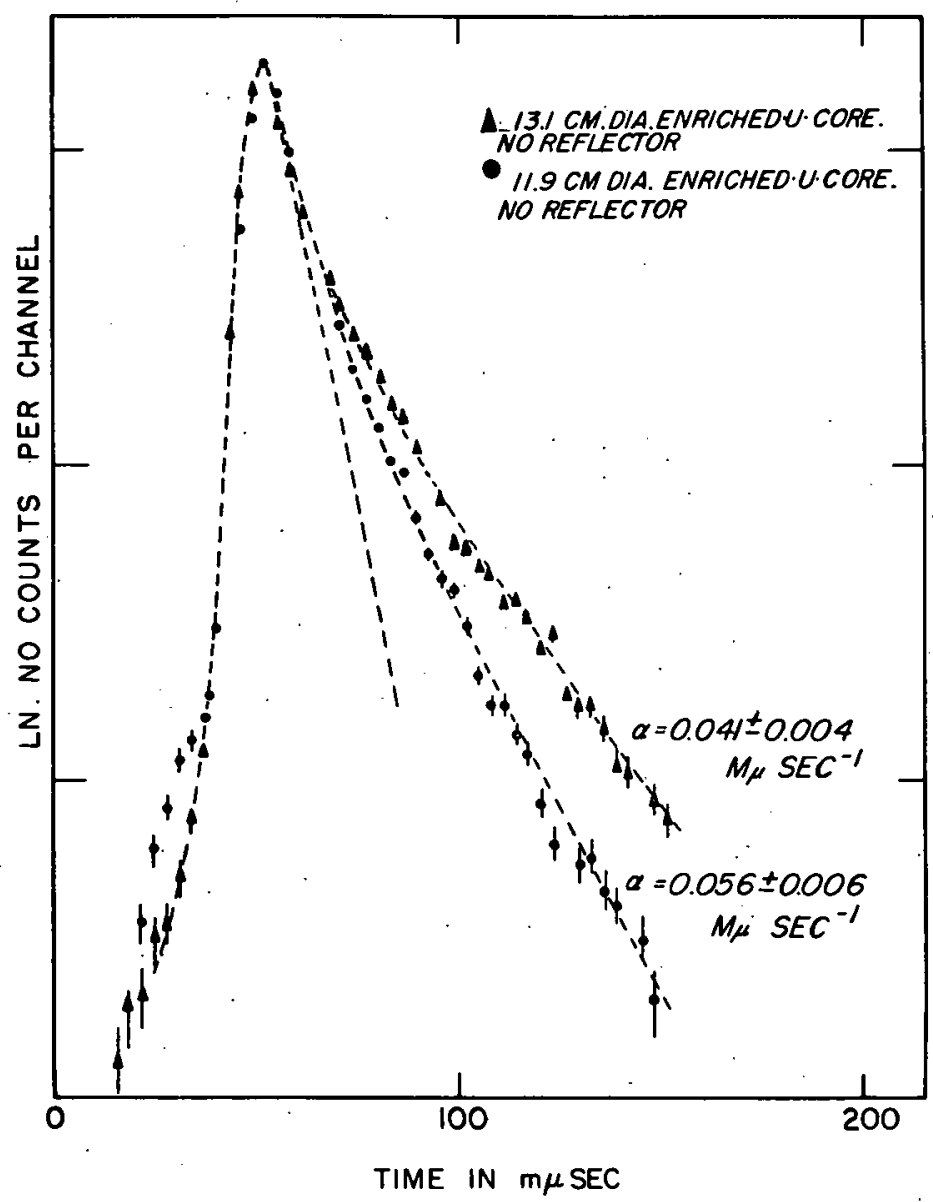

MUL. 2604

Fig. 2. Experimentally observed curves for $13.1-\mathrm{cm}$ and $11.9-\mathrm{cm}$ diameter enriched-uranium spheres. Neutron burst shown as dashed line.
Fig. 1. Comparison of Eqs. (8a) and (8b) with experiment $-13.1-\mathrm{cm}$ diameter enriched-uranium sphere. 
$-11-$

UCRL-4808

APPENDIX

Solution to Ens. (1) and (2), with $S(t)$ Given by Eqs. (ja) and (Bb)

i) $0 \leq \mathrm{t} \leq \tau$

$$
\begin{aligned}
& n(t)=\frac{1}{\left(\rho_{1}-\rho_{2}\right)}\left[\left(\rho_{1}+\lambda\right) n(0)+\lambda c(0)-\frac{S_{0} \gamma\left(\rho_{1}+\lambda\right)}{\left(e^{\gamma \cdot \tau}-1\right)\left(\gamma-\rho_{1}\right) \rho_{1}}\right] e^{\rho_{1} t} \\
& +\frac{S_{0}}{\left(e^{\gamma \tau}-1\right)}\left[\frac{(\gamma+\lambda) e^{\gamma t}}{\left(\gamma-\rho_{1}\right)\left(\gamma-\rho_{2}\right)}-\frac{\lambda}{\rho_{1} \rho_{2}}\right] \\
& -\frac{1}{\left(\rho_{1}-\rho_{2}\right)}\left[\left(\rho_{2}+\lambda\right) n(0)+\lambda c(0)-\frac{S_{0} \gamma\left(\rho_{2}+\lambda\right)}{\left(e^{\gamma / \tau}-1\right)\left(\gamma-\rho_{2}\right) \rho_{2}}\right] e^{\rho_{2}} \\
& c(t)=\frac{1}{\left(\rho_{1}-\rho_{2}\right)}\left\{\frac{\beta k_{\text {eff }}}{\ell} n(0)+\left[\rho_{1}+\frac{1-k_{e f f}^{i}(1-\beta)}{\ell}\right] c(0)\right. \\
& \left.\therefore \frac{s_{0} \gamma \beta k_{e f f}}{\left(e^{\gamma \tau}-1\right)\left(\gamma-\rho_{1}\right) \rho_{1} \ell}\right\} e^{\rho_{I}^{t}}+\frac{\beta k_{\text {eff }}}{\left(e^{\gamma \tau}-1\right) \ell}\left[\frac{e^{\gamma t}}{\left(\gamma-\rho_{1}\right)\left(\gamma-\rho_{2}\right)}-\frac{1}{\rho_{1} \rho_{2}}\right] \\
& -\frac{1}{\left(\rho_{1}-\rho_{2}\right)}\left\{\frac{\beta k_{e f f}}{\ell} n(0)+\left[\rho_{2}+\frac{1-k_{e f f}(1-\beta)}{l}\right] c(0) \frac{s_{0} \gamma \beta k_{e f f}}{\left(e^{\gamma \tau}-1\right)\left(\gamma-\rho_{2}\right) \rho_{2} l}\right\} e^{\rho_{2}}{ }^{t} \\
& \text { iii) } \tau \leq \mathrm{t} \\
& n(t)=\frac{1}{\left(\rho_{1}-\rho_{2}\right)}\left[\left(\rho_{1}+\lambda\right) n(0) e^{\rho_{1} \tau}+\lambda c(0) e^{\rho_{1} \tau}-\frac{s_{0} \gamma\left(\rho_{1}+\lambda\right)\left(e^{\rho_{1} \tau}-1\right)}{\left(e^{\gamma \tau}-1\right)\left(\gamma-\rho_{1}\right) \rho_{1}^{\prime}}\right. \\
& \left.+\frac{2 S_{0}\left(\rho_{1}+\lambda\right) \gamma}{\left(\gamma+\rho_{1}\right)\left(\gamma-\rho_{1}\right)}\right] e^{\rho_{1}(t-\tau)}-\frac{\dot{S}_{0}(\gamma-\lambda) e^{\gamma(t-\tau)}}{\left(\gamma+\rho_{1}\right)\left(\gamma+\rho_{2}\right)}-\frac{1}{\left(\rho_{1}-\rho_{2}\right)}\left[\left(\rho_{2}+\lambda\right) n(0) e^{\rho_{2} \tau}\right. \\
& 738
\end{aligned}
$$


$-12-$

UCRL -4808

$$
\left.+\lambda c(0) e^{\rho_{2} \tau}-\frac{S_{0} \gamma\left(\rho_{2}+\lambda\right)\left(e^{\rho_{2} \tau}-1\right)}{\left(e^{\gamma \tau}-1\right)\left(\gamma-\rho_{2}\right) \rho_{2}}+\frac{2 S_{0}\left(\rho_{2}+\lambda\right) \gamma}{\left(\gamma+\rho_{2}\right)\left(\gamma-\rho_{2}\right)}\right] e^{\rho_{2}(t-\tau)}
$$

$$
c(t)=\frac{1}{\left(\rho_{1}-\rho_{2}\right)}\left\{\frac{\beta_{\text {eff }}}{\ell} n(0) e^{\rho_{1} \tau}+\left[\rho_{1}+\frac{1-k_{e f f}(1-\beta)}{l}\right] c(0) e^{\rho_{1} \tau}\right.
$$

$$
\left.-\frac{S_{0} \gamma \beta k_{e f f}\left(e^{\rho_{1} \tau}-1\right)}{\left(e^{\gamma \cdot \tau}-1\right)\left(\gamma-\rho_{1}\right) \rho_{1} \ell}+\frac{2 S_{0} \beta k_{e f f} \gamma}{\left(\gamma+\rho_{1}\right)\left(\gamma-\rho_{1}\right) \ell}\right\} e^{\rho_{1}(t-\tau)}+\frac{S_{0} \beta k_{e f f}}{\left(\gamma+\rho_{1}\right)\left(\gamma+\rho_{2}\right)} \cdot e^{-\gamma(t-\tau)}
$$

$$
\begin{aligned}
& -\frac{1}{\left(\rho_{1}-\rho_{2}\right)}\left\{\frac{\hat{\beta k}_{\text {eff }}}{\ell} \mathrm{n}(0) \mathrm{e}^{\rho_{2} \tau}+\left[\rho_{2}+\frac{1-k_{\text {eff }}(1-\beta)}{\ell}\right] c(0) \mathrm{e}^{\rho_{2} \tau}\right. \\
& \left.-\frac{\mathrm{S}_{0} \gamma \beta \mathrm{k}_{\mathrm{eff}}\left(\mathrm{e}^{\rho_{2} \tau}-1\right)}{\left(\mathrm{e}^{\gamma \tau}-1\right)\left(\gamma-\rho_{2}\right) \rho_{2} \ell}+\frac{2 \mathrm{~S}_{0} \beta \mathrm{k}_{\mathrm{eff}} \gamma}{\left(\gamma+\rho_{2}\right)\left(\gamma-\rho_{2}\right) \ell}\right\} \mathrm{e}^{\rho_{2}(\mathrm{t}-\tau)},
\end{aligned}
$$

7

where

$$
\begin{aligned}
& \rho_{1}=1 / 2\left\{-\left[\lambda+\frac{1-k_{\text {eff }}(1-\beta)}{\ell}\right]-\sqrt{\left[\lambda-\frac{1-k_{e f f}(1-\beta)}{\ell}\right]^{2}+\frac{4 \lambda \beta k_{\text {eff }}}{\ell}}\right\} \\
& \rho_{2}=1 / 2\left\{-\left[\lambda+\frac{1-k_{\text {eff }}(1-\beta)}{\ell}\right]+\sqrt{\left.\left[\lambda-\frac{1-k_{\text {eff }}(1-\beta)}{\ell}\right]^{2}+\frac{4 \lambda \beta k \text { eff }}{\ell}\right\}}\right.
\end{aligned}
$$

In the systems of interest

$$
\left[\lambda-\frac{1-k_{\text {eff }}(1-\beta)}{\ell}\right]^{2}>\frac{4 \lambda \beta k_{\text {eff }}}{\ell}
$$

hence $\quad \rho_{1} \approx-\lambda \quad$ and $\quad \rho_{2} \approx \frac{1-k_{\text {eff }}(1-\beta)}{\ell}$.

$73 b$

12 